\title{
Cancer cells' low sensitivity to oxalate
}

Xiaoxia Li $^{2, *}$, Tianlun Lei ${ }^{1, *}$, Yilin Zhang ${ }^{1}$, Man Tang ${ }^{1}$, Meng Xing ${ }^{1}$, Jingli Peng ${ }^{1}$, Min $\mathrm{Fu}^{1}, \mathrm{Li} \mathrm{Xu}{ }^{1}$, Wenliang Zhou ${ }^{1}$, Qiuyun Liu ${ }^{1, * *}$

${ }^{1}$ School of Life Sciences, Sun Yat-sen University, Guangzhou 510275, China.

${ }^{2}$ School of Materials Science and Engineering, Sun Yat-sen University, Guangzhou 510275, China.

*Xiaoxia Li and Tianlun Lei contributed equally to this work.

${ }^{* *}$ Correspondence: Qiuyun Liu, Ph.D, School of Life Sciences, Sun Yat-sen University, Guangzhou 510275, China. Tel: [+86 (020) 84110296]; E-mail: 1sslqy@mail.sysu.edu.cn 


\begin{abstract}
The Warburg effect refers to the phenomenon that cancer cells produce energy via glycolysis instead of cellular respiration. Glycolysis generated no net protons. The Warburg effect may be malignant cells' built-in mechanism to antagonize the buildup of protons via Krebs cycle and other pathways with compromised cellular respiration. Data described in this study indicated that cancer cells were less sensitive to the presence of oxalate than non-cancer model cell lines 16HBE14o- and HaCaT. Malignant cells may resort on organic acids such as oxalate and their calcium salts to antagonize strong acids. This experiment sheds light on the role of Warburg effect in cancer cell metabolism and homeostasis.
\end{abstract}

\title{
Key words
}

Warburg effect; Protons; Calcium oxalate; Antagonization 
1

\section{Introduction}

The Warburg effect describes the phenomenon that most cancer cells predominantly rely on glycolysis instead of cellular respiration for energy generation [1]. A hypothesis proposed that the local buildup of strong acids such as $\mathrm{HCl}$ is the major risk factor of carcinogenesis [2-3]. The Krebs cycle is a principle source of protons and organic acids, whose accumulation can be triggered by the impaired respiratory chain. Glycolysis generated no net protons, and the coupled gluconeogenesis as well as the Krebs cycle produce oxaloacetate which is metabolized in cells to form oxalate. The divalent salts of oxalate such as calcium oxalate are highly insoluble and rigid, conferring stresses to living cells [4-5]. Compounds such as ethanol and acetic acids are structurally similar to oxalate, and can extend lifespan [6-7], perhaps via the inhibition of oxalate generation. Glycolic acid used in skin-care products helps remove age-related wrinkles. Yet, weak or moderate strength organic acids have a positive side as they can counteract strong acids. Numerous Chinese vinegar factories reported few cancer cases over decades as volatile acetic acid may antagonize $\mathrm{HCl}$ [8-10]. Calcium supplement substantially reduces cancer risks due to its neutralization of strong acids [2].

\section{Materials and methods}

Flow cytometry.

The apoptosis and necrosis of various kinds of cells were analyzed using the Annexin V-FITC/PI staining Kit (KeyGEN, China).[11] After incubations for $24 \mathrm{~h}$ in the presence or absence of $2 \mathrm{mM}$ sodium oxalate respectively, the cells were digested by using $0.25 \%$ trypsin and then collected, followed by washing twice with fresh PBS. $300 \mu$ l Binding Buffer was added to resuspend the cells which were then labeled with AnnexinV-FITC $(5 \mu \mathrm{l})$ and PI $(5 \mu \mathrm{l})$ in the dark for $15 \mathrm{~min}$. Fluorescence intensities were detected using a Beckman Coulter Gallios Flow Cytometer, with $525 \mathrm{~nm}$ excitation in FL1 for AnnexinV-FITC and $575 \mathrm{~nm}$ excitation in FL2 for PI. Acquired data were analyzed with Kaluza software. 


\section{Results and discussion}

Cancer cells were relatively insensitive to the presence of oxalate compared to non-cancer normal model cell lines 16HBE14o- and HaCaT (Fig 1), particularly A549 human lung cancer cells. The percentages of apoptotic and necrotic cells in normal 16HBE14o- human bronchial epithelial cells with or without oxalate were $30.81 \%$ and $8.16 \%$ respectively. The percentages of apoptotic and necrotic cells in normal HaCaT Keratinocytes, BEL-7402 human liver cancer cells and A549 human lung cancer cells with or without oxalate were $30.08 \%, 7.00 \% ; 22.90 \%$, $4.61 \% ; 11.63 \%, 5.26 \%$ respectively. Therefore, malignant cells might take advantage of this selective edge for survival, and outcompete normal cells.

The excessive oxalate levels generated in cancer cells may spread to adjacent areas and confer stresses to surrounding normal cells, and the buildup of insoluble and rigid calcium oxalate causes death of the normal cells and the patients [12]. The renal calcium oxalate stones sometimes lead to kidney failure. Oxalate levels were higher in breast cancer cells than their corresponding non-pathological breast tissue in a previous report [13]. Various approaches can be adopted to minimize the generation of oxalate, such as the use of structurally similar acetic acid and lactic acid as inhibitors of oxalate production [9-10]. Since the overconsumption of $\mathrm{NaCl}$ gives rise to $\mathrm{HCl}$ occasionally, weak organic acids free of salts can also be used to reduce cancer risks [10]. RNAi technology can be harnessed to reduce oxalate production to avoid detrimental effects on cancer patients [14]. 


\section{Conflict of interest}

None declared.

\section{Acknowledgments}

This work was supported by grants from the Science and Technology Transformation Program of Sun Yat-sen University of China (33000-18843234), Guangzhou Science and Technology Program (201804010328); Guangdong Science and Technology Program (2016B020204001, 2008B020100001); Guangdong Natural Science Foundation (S2011010004264) and The National Natural Science Foundation of China (30370799) to Q. Liu. We are grateful to Jiaming Zhang, Weiguo Cao and Yuchuan Wang for discussions and suggestions. Editing by Yan Shi is appreciated.

\section{References}

1. Alfarouk KO, Verduzco D, Rauch C, et al (2014) Glycolysis, tumor metabolism, cancer growth and dissemination. A new pH-based etiopathogenic perspective and therapeutic approach to an old cancer question. Oncoscience 1:777-802.

2. Tang M, Zhou Y, Li Y, et al (2017) Hydrogen donors and acceptors and basic amino acids jointly contribute to carcinogenesis. Med Hypotheses 98:42-44.

3. Zhang J, Tang M, Shi Y, Wan Y, Hu X, Liu Q (2017) Appearance of somatic mutations can be subjected to the influences of inherited factors. Science. http://science.sciencemag.org/content/355/6331/1330/tab-e-letters.

4. Gao Y, Huang Y, Dai D, Wu X, Yang W, Liu Q (2017) Organic acids and calorie restriction. Science. http://science.sciencemag.org/content/350/6265/1204/tab-e-letters.

5. Li J, Li Z, Feng F, Shi Y, Liu Q (2018) Calcium supplement is a major concern for patients with cardiovascular diseases. Eur J Prev Cardiol 25:641.

6. Chuang MH, Chiou SH, Huang CH, Yang WB, Wong CH (2009) The lifespan-promoting effect of acetic acid and Reishi polysaccharide. Bioorg Med Chem 17:7831-7840. 
7. Paganini-Hill A, Kawas CH, Corrada MM, et al (2007) Type of alcohol consumed, changes in intake over time and mortality: the Leisure World Cohort Study. Age Ageing 36:203-209.

8. Shi Y, An S, Wan Y, Yang F, Liu Q (2019) How to best use acetic acid for the prevention of heart disease and cancer. Eur J Prev Cardiol 26(4):437-438. DOI: $10.1177 / 2047487318784356$.

9. An S, Wan Y, Shi Y, Yang F, Liu Q (2018) How to choose medicinally more valuable yogurt products for the prevention of heart disease and colorectal cancer. Eur J Prev Cardiol 25(18):2013-2014. DOI: 10.1177/2047487318784994

10. Wan Y, Hu X, Yan S, Liu Q (2018) NaCl-free daily meals may be essential to maximize the health benefits of the acetic acid-based cancer therapies. Science. http://science.sciencemag.org/content/355/6331/1330/tab-e-letters

11. An S, Li X, Tang M, et al (2020) The role of acetate in the antagonism of oxalate - A potential causative molecule for heart disease and cancer death. Nat Prod Commun 15(3):1-4. doi.org/10.1177/1934578X20913697

12. He J, Weng S, Lai X, Liu Q (2017) Oxalate may be a death mediator. Science. http://science.sciencemag.org/content/350/6265/1204/tab-e-letters

13. Castellaro AM, Tonda A, Cejas HH, et al (2015) Oxalate induces breast cancer. BMC Cancer 15:761.

14. Du Y, Fu M, Peng J, An S, Liu Q (2018) How to alleviate cancer-caused secondary heart disease. Eur J Prev Cardiol 25(15):1675. DOI: $10.1177 / 2047487318782510$. 

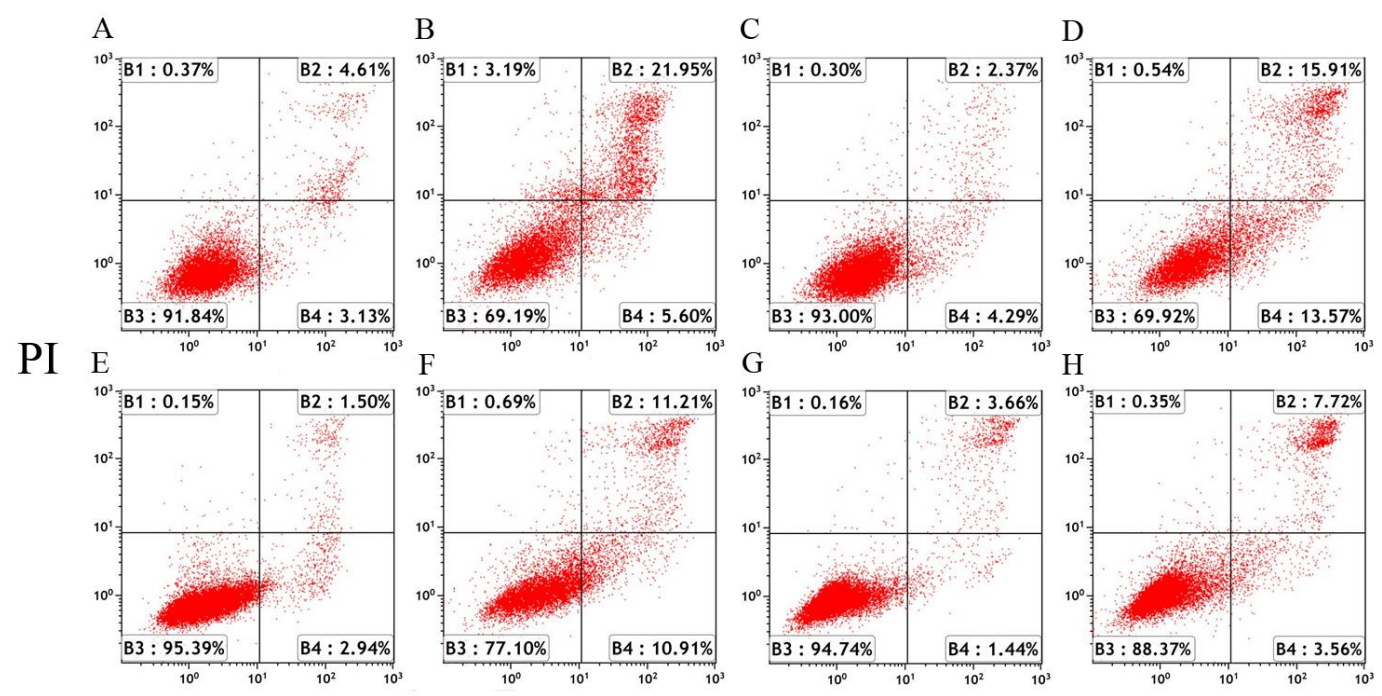

Annexin V-FITC

Fig. 1. Quantitation of cell apoptosis of various cell lines using Annexin V-FITC/PI staining via flow cytometry. The percentages of normal (B3 quadrant), early apoptotic (B4 quadrant), late apoptotic (B2 quadrant) and necrotic (B1 quadrant) cells were measured $24 \mathrm{~h}$ after treatments with $2 \mathrm{mM}$ sodium oxalate. (A,B) (16HBE14o- human bronchial epithelial cells: without oxalate, with sodium oxalate. c to h likewise), (C,D)

111 (HaCaT Keratinocytes), (E,F) (BEL-7402 human liver cancer cells), (G,H) (A549 human lung cancer cells). Pearson $\chi^{2}$ significances for differences on apoptosis and necrosis between the cancer cells and the cells of non-cancer model cell lines in the presence or absence of oxalate were 0.000 respectively (two tailed, SPSS 22.0). 